\title{
Some aspects regarding the influence of the anisotropy of an AA2021-T351 rolled thick plate on its tribological behaviour
}

\author{
Elisabeta Pirva ${ }^{1}$, Andrei Tudor ${ }^{2}$, Adinel Gavrus ${ }^{3,}$, Nicolae Stoica ${ }^{2}$, and Sorin Cananau ${ }^{2}$ \\ ${ }^{1}$ University Politehnica of Bucharest (UPB), 313 Splaiul Independentei, 060042 Bucharest, Romania \\ ${ }^{2}$ University Politehnica of Bucharest (UPB), OMT Department, 313 Splaiul Independentei, 060042 Bucharest, Romania \\ ${ }^{3}$ Institut National des Sciences Appliquées de Rennes (INSA Rennes), Laboratoire de Génie Civil et Génie Mécanique \\ (LGCGM) EA 3913, University Brittany Loire (UBL), 20 av. des Buttes de Coesmes, 35708 Rennes, France
}

Received: 23 January 2019 / Accepted: 6 September 2019

\begin{abstract}
This scientific paper aims to study the influence of the anisotropy of an aluminium AA2024-T251 rolled thick plate on the surface tribological properties based on previous experimental and numerical studies concerning bulk and surface mechanical behaviour. Experimental friction tests have been made on the aluminium alloy thick plate surface using sliding of an ultra-high-molecular-weight polyethylene (UHMWPE) cylindrical pin. The used tribological test involves the generation of local motion along specified trajectories with different orientations under the action of a constant normal force and a constant slip velocity. Three different normal forces $(3 \mathrm{~N}, 5 \mathrm{~N}, 7 \mathrm{~N})$ have been applied using five different sliding speeds $(0.005,0.05,0.5,1 \mathrm{and} 5 \mathrm{~mm} / \mathrm{s})$ along a linear trajectory of $3 \mathrm{~mm}$ length. In order to analyze the influence of the aluminium alloy anisotropy, the tests have been performed along three directions: a longitudinal one, corresponding to the rolling direction of the sample $\left(0^{\circ}\right)$, a transverse one, perpendicular to the rolling direction $\left(90^{\circ}\right)$, and a median direction $\left(45^{\circ}\right)$. Taking into account the observed bulk and surface anisotropy, especially concerning a fractal nature of the surface topography, an investigation was performed in order to determine its influence on the anisotropic tribological properties. In this purpose, fractal friction characteristics have been determined for the sliding process using a constant normal force $F_{n}=7 N$ and the above five sliding speeds.
\end{abstract}

Keywords: Anisotropy / tribology / aluminium alloy / anisotropic friction behaviour / friction fractal analysis

\section{Introduction}

Friction has an important role in many industrial manufacturing processes and the last developments of numerical engineering design tools require reliable analyses of friction phenomena and rigorous identification of material's tribological properties. The application of low weight materials as aluminium alloys and plastics used by the automotive, railways and aeronautics industry has grown since their introduction in the mid-1970s. During the past three decades a lot of metallic-plastics structures joints, mechanical assemblies and couplings, particularly defined by aluminium alloy-plastic interfaces have been designed (Fig. 1).

The contact area corresponding to these types of materials couples undergoing generally a dry or a boundary lubrication friction, a slow speed sliding and an intermittent moving within range limits of temperature. Many plastics are even self-lubricating, have an extremely

\footnotetext{
* e-mail: adinel.gavrus@insa-rennes.fr
}

corrosive resistance, a high chemically resistance and therefore avoid the possibility of failures from lack of maintenance. The most commonly used in industry are the phenolics, the acetals, the teflon (PTFE), the ultra-high molecular weight polyethylene (UHMWPE) and the nylon [1]. In most sliding or rolling kinetics during a material forming process the friction causes loss energy and high material's wear (forging, extrusion, cutting, etc) and in these cases, friction should be minimized (forging - low friction along the rib). There are a lot of cases where friction has a driving role (rolling - need of a minimum friction, forging - increased friction on the outer flat) or where an optimal friction is required (deep drawing, stretch forming process) [2]. It is known today that the friction between two solid surfaces is a very complex phenomenon that depends not only on the physical and chemical properties of the involved materials, but also on the thermo-mechanical properties of the contact surfaces. All the investigations of friction are mostly based on experimental, theoretical and numerical studies. Depending on the nature of the materials and of the experimental conditions, the general evolution of friction forces can be presented in Figure 2 [3]. 
Table 1. Elasto-plastic parameters of the anisotropic aluminium alloy AA2024-T351 [9-11].

\begin{tabular}{|c|c|c|c|c|c|c|c|c|c|}
\hline \multicolumn{2}{|c|}{$\begin{array}{l}\text { Elasticity } \\
\text { parameters }\end{array}$} & \multicolumn{3}{|c|}{$\begin{array}{l}\text { Yield stress [MPa] } \\
\left(\bar{\varepsilon}^{p}=0.2 \%\right)\end{array}$} & \multicolumn{2}{|c|}{$\begin{array}{l}\text { Voce law }\left(0^{\circ}\right) \\
\sigma\left(0^{\circ}\right)=\sigma_{0}\left(0^{\circ}\right)+K\left[1-\exp \left(-n \bar{\varepsilon}^{p}\right)\right]\end{array}$} & \multicolumn{3}{|c|}{ Lankford coefficients } \\
\hline$E$ [MPa] & $v$ & $\sigma_{0}\left(0^{\circ}\right)$ & $\sigma_{0}\left(45^{\circ}\right)$ & $\sigma_{0}\left(90^{\circ}\right)$ & $K[\mathrm{MPa}]$ & $n$ & $r_{0}$ & $r_{45}$ & $r_{90}$ \\
\hline 72653 & 0.33 & 301.0 & 266.0 & 250.0 & 250.0 & 18.0 & 0.61 & 0.73 & 0.59 \\
\hline
\end{tabular}

a)
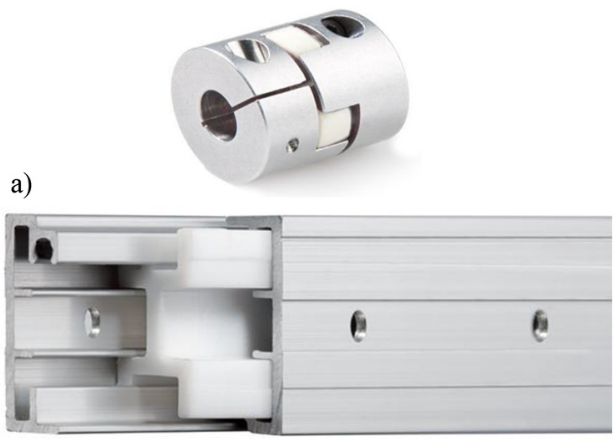

b)

Fig. 1. Examples of aluminium alloy-plastics interfaces. (a) Jaw coupling; (b) Telescopic sliding system.

Under a normal force $F_{n}$, the Coulomb coefficient of static friction $\mu_{s}$ corresponding to a tangential force $F_{f}=F_{s}$ is distinct from the Coulomb coefficient of kinetic friction $\mu_{k}$ corresponding to $F_{f}=F_{k}$ as is illustrated in Figure $2 \mathrm{a} . F_{s}$ is the maximum tangential force required to have a motion of solid, and $F_{k}$ is the force applied to maintain this motion [4]:

$$
F_{s}=\mu_{s} F_{n} \quad F_{k}=\mu_{k} F_{n}
$$

The classical stick-slip (shown in Fig. 2b) requires a static friction coefficient greater than the kinetic friction coefficient. In the case of sliding surfaces, the time period of the contact between two points on the two surfaces is longer when the surfaces slide slowly than when they move rapidly. If the sliding of one surface over another slows down, friction increases. This is the situation that favours the stick-slip. The phenomenon appears in metallic friction couples with dry or limited friction regime, when the sliding speed is in the range of $0.01-3 \mathrm{~mm} / \mathrm{s}$ or when the angular speed is somewhere in the $1-25 \mathrm{rad} / \mathrm{s}$ range. Studies of sliding at slow speeds are important because they provide systematic information about the friction-velocity relationships required by the engineering designers to select materials that will not be affected by stick-slip phenomenon. Three main classical methods can be used to preserve or reduce stick-slip phenomenon: changing the sliding speed (in some cases slowing down, in some cases speeding up), reducing the stored energy or lubricating the sliding surfaces $[5,6]$. The main purpose of this work is to analyze the rheological and tribological behaviour especially regarding the influence of the anisotropic properties on the metallic-plastic interfaces.
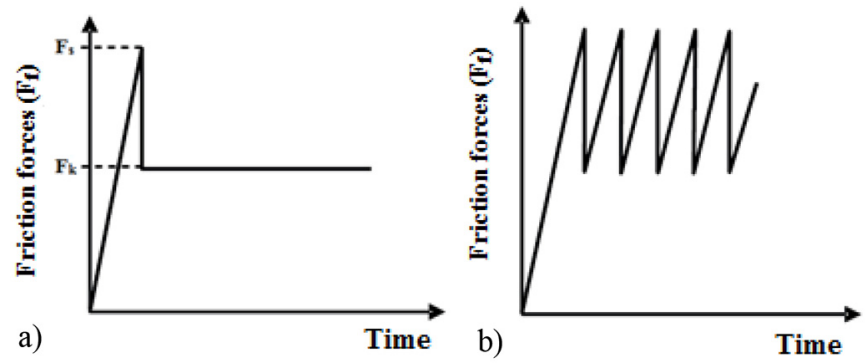

Fig. 2. Evolution of the friction force $v s$ time. (a) sliding without stick-slip; (b) stick-slip sliding [3].

\section{Anisotropic material properties}

Aluminium alloys are widely used in many industrial fields such as aeronautics, naval, railway and automotive. The most considerable particularities of aluminium alloys are their good ductility (especially at high temperatures), high electrical conductivity, low specific weight and their very good formability and workability. Because of these excellent thermo-mechanical and physical characteristics, the usage of aluminium has an ever-increasing importance in a lot of industrial manufacturing industry: rolling, extrusion, molding, embossing, stamping and welding [7]. This paper proposes to analyze bulk and surface anisotropic properties of an aluminium alloy AA2024-T351 rolled thick plate with a thickness of $10 \mathrm{~mm}$. The main mechanical properties of this aluminium alloy are defined by an ultimate tensile strength higher than $420 \mathrm{MPa}$, a $\mathrm{HB}$ hardness around $120 \mathrm{Kgf} / \mathrm{mm}^{2}$ and a yield strength at $0.2 \%$ greater than $260 \mathrm{MPa}$. Experimental and numerical identification of anisotropic elasto-plastic parameters are shown in Tables 1 and 2, starting from previous experimental, theoretical and numerical works [9-11] concerning the analysis of bulk plastic anisotropic behaviour realized by GCGM Laboratory of National Institute of Applied Sciences of Rennes.

In the case of a rolled sheet is generally assumed to have an orthotropic plastic anisotropy considering three specific material orientations: $x$-rolling direction $\operatorname{LD}\left(0^{\circ}\right), y$ transverse direction TD $\left(90^{\circ}\right)$ and $z$-thickness direction. The plane and normal anisotropy are analyzed from the estimation of the Lankford coefficient $r$ defined by the ratio between the transversal plastic strain rate and the thickness plastic strain rate. Experimental tensile tests have been performed using different loading orientations 
Table 2. Experimental and numerical identified Hill's 1948 coefficients of AA2024-T351 thick plate [9-11].

\begin{tabular}{lllll}
\hline Hill parameters $(\mathrm{MPa})$ & $F$ & $G$ & $H$ & $L=M=N$ \\
\hline Experimental plane anisotropy $\left(\bar{\varepsilon}^{p}=12 \%\right)$ & 0.64 & 0.62 & 0.38 & 1.74 \\
Experimental normal anisotropy $\left(\bar{\varepsilon}^{p}=12 \%\right)$ & 0.60 & 0.60 & 0.40 & 1.40 \\
Numerical identification of plane anisotropy & 0.50 & 0.63 & 0.37 & 0.97 \\
Numerical identification of normal anisotropy & 0.63 & 0.63 & 0.36 & 1.35 \\
\hline
\end{tabular}

with respect to the rolling direction $\left(0^{\circ}, 45^{\circ}\right.$ and $\left.90^{\circ}\right)$ for a lot of specific AA20124-T351 specimens with a thickness of $3 \mathrm{~mm}$. The corresponding experimental values of Lankford coefficients $r_{0}, r_{45}, r_{90}$ have been computed from measurements of local longitudinal and transversal plastic strain rate corresponding to different plastic deformation degrees and especially for an equivalent plastic strain equal to $12 \%$ characterising the beginning of diffuse striction (Tab. 1). According to the Lankford analysis and using specific hypothesis, dimensionless coefficients have been identified for a Hill'48 yield anisotropic criterion (Tab. 2):

$$
\begin{aligned}
\Phi([\sigma])= & F\left(\sigma_{y y}-\sigma_{z z}\right)^{2}+G\left(\sigma_{z z}-\sigma_{x x}\right)^{2} \\
& +H\left(\sigma_{x x}-\sigma_{y y}\right)^{2}+2 L \tau_{y z}^{2} \\
& +2 M \tau_{z x}^{2}+2 N \tau_{x y}^{2}-\sigma^{2}\left(0^{\circ}\right)=0 .
\end{aligned}
$$

In the case of a plane anisotropy behaviour (generally used to describe anisotropy of thin sheets) if a reference equivalent stress along the rolling direction $\left(0^{\circ}\right)$ is considered $G+H=1$ and consequently only three independent Hill parameters $F, G$ and $N$ can be estimated using specific relationships expressed in terms of Lankford coefficients [11]. For a thick plate a general orthotropic three-dimensional plastic anisotropy must be defined. In a first approximation if the experimental planar anisotropy coefficient has a value closed to 0 i.e. $\Delta \bar{r} \approx 0$ (here $\Delta \bar{r}=-0.06)$ a normal anisotropy can be used. In this case $F=G$ and $N=F+2 H$ where the shear anisotropic parameters $L$ and $M$ are keep equal to $N$, i.e. $L=M=N$. Beside a first experimental estimation based on classical Lankford coefficients evaluation method, the Hill parameters have been also identified numerically by finite element simulations [10-11] starting from an interactivegraphic non-linear regression of the true stress- true plastic strain curves obtained from tensile tests along $0^{\circ}, 45^{\circ}$ and $90^{\circ}$. A Voce hardening law has been used to define a reference equivalent stress from the tensile curve obtained for a loading along the rolling direction $\left(0^{\circ}\right)$. The identified anisotropic Hill criteria describe the bulk anisotropic mechanical behaviour and the macroscopic surface stress state of the elasto-plastic contact interfaces.

\section{Experimental tribological procedure}

As specific part of this scientific work a tribological analysis of the aluminium alloy AA2024-T351 rolled thick plate with a thickness of $10 \mathrm{~mm}$ has been performed from pin on

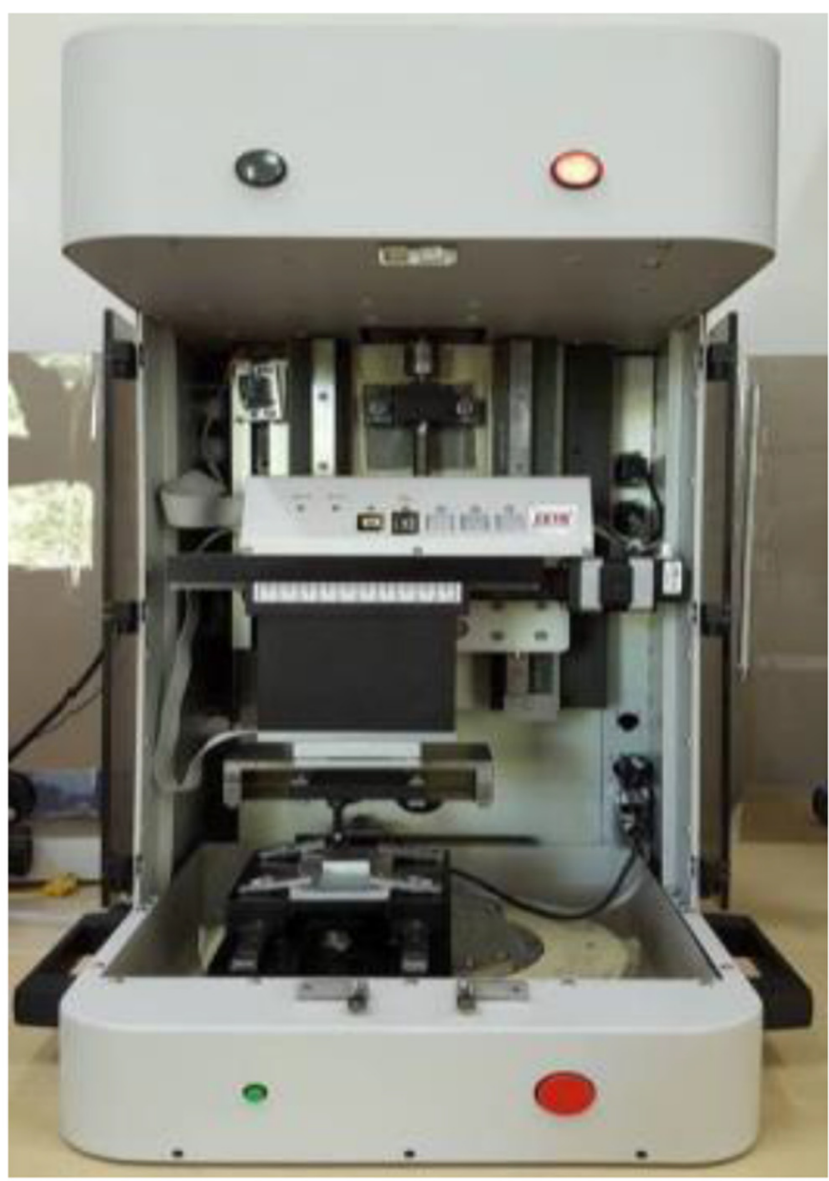

Fig. 3. UMT Tribometer.

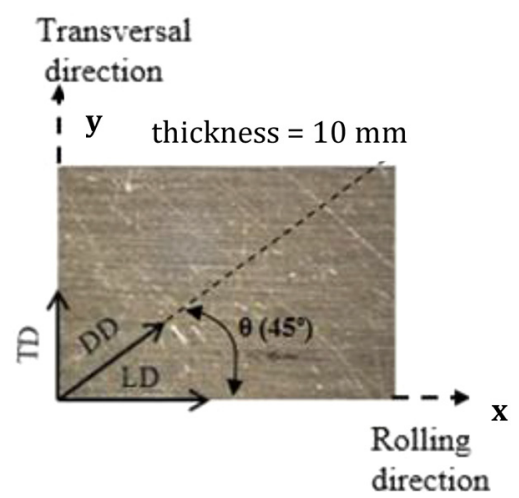

Fig. 4. Measuring directions (rolled AA2024-T351 plate). 


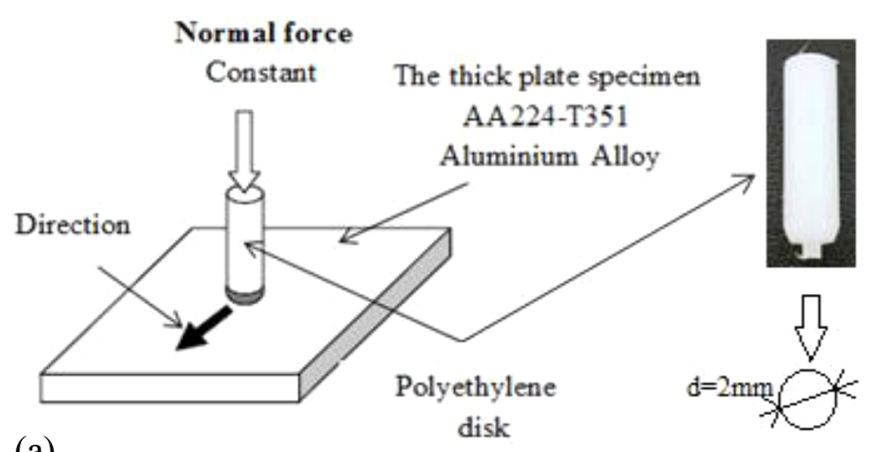

(a)

Fig. 5. Friction test conditions. (a) Tribometer schema; (b) Surface micro-topography.

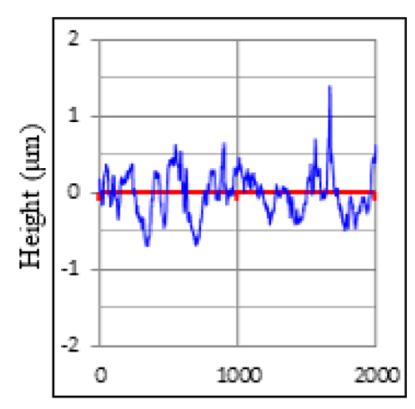

(b) Length $(\mu \mathrm{m})$ plan translation friction tests using an ultra-high-molecular-weight polyethylene (UHMWPE) cylindrical pin with a diameter of $2 \mathrm{~mm}$. This polymer is characterized by an excellent resistance to abrasion and has particular mechanical properties defined by an ultimate tensile strength around $38.6 \mathrm{MPa} \div 48.3 \mathrm{MPa}$, a yield strength of $21.4 \mathrm{MPa} \div 27.6 \mathrm{MPa}$, a Young's modulus $E=894 \mathrm{MPa}$ $\div 963 \mathrm{MPa}$ and a Poisson's coefficient of 0.46 [8]. The used UMT Tribometer (OMT Department of UPB) is presented in Figure 3.

This tribometer can provide rotation (pin on disk), translation (pin on plan) or reciprocating motions with speeds starting from $0.1 \mu \mathrm{m} / \mathrm{s}$ up to $10 \mathrm{~m} / \mathrm{s}$. A constant or progressive load between 0.05 and $1000 \mathrm{~N}$ can be applied to the contact surfaces [12]. In order to analyze the influence of the bulk and surface material anisotropy, dry sliding tribological tests were made on three particular directions (Fig. 4): a longitudinal one $\mathrm{LD}\left(0^{\circ}\right)$, corresponding to the rolling direction of the sample, a transversal one TD $\left(90^{\circ}\right)$ which represents the direction perpendicular to the rolling direction and a median direction DD $\left(45^{\circ}\right)$.

The tests involve moving a polyethylene cylindrical pin along a specified trajectory under a constant normal force and at a constant speed (Fig. 5a).

All the tribological tests were performed for three different normal forces $(3 \mathrm{~N}, 5 \mathrm{~N}, 7 \mathrm{~N})$ and five different speeds $(0.005,0.05,0.5,1$ and $5 \mathrm{~mm} / \mathrm{s})$ along a linear trajectory with a length around $3 \mathrm{~mm}$ for the smallest velocities and around $30 \mathrm{~mm}$ for the higher ones (1 and $5 \mathrm{~mm} / \mathrm{s}$ ).

Starting from previous works of authors [13-14] where micro-topography of the rolled aluminium alloy AA2024T351 surface was studied, the random nature of the roughness height has been described through statistical and fractal analysis. Roughness is one of the main characteristics of surface quality and it can be determined by special contact profilometers (Fig. 5b) and by noncontact measurement methods such as optical scanning of the surface shape (Fig. 6).

A specific roughness structural function method has been used to compute the corresponding geometric fractal dimension $D_{f}[13]$. It was observed that the samples surface have a fractal behaviour up to $1.1 \mu \mathrm{m}$. The estimated values of $D_{f}$ and the corresponding variation curves have shown that the effect of the fractal dimension are smaller

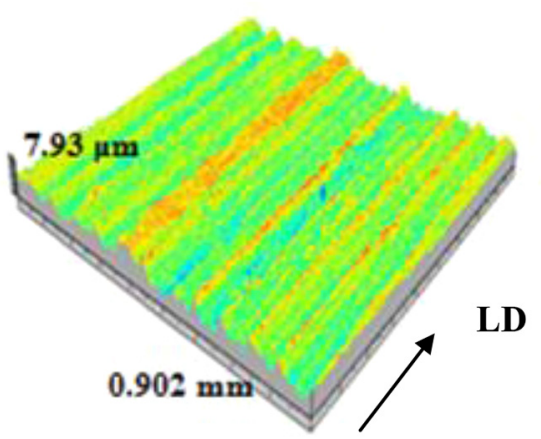

Fig. 6. 3D Optical surface profilometry.

for the longitudinal direction and that the fractal dimensions have similar values for transversal and median directions.

\section{Anisotropic friction analysis}

The time variation of the tangential forces $F_{f}$ for all measurements corresponding to three normal forces $F_{n}$ $(3 \mathrm{~N}, 5 \mathrm{~N}, 7 \mathrm{~N})$ and five different sliding speeds $v_{g}$ are presented in Figure 7.

Because of the polyethylene semi-crystalline nature, which has excellent abrasion resistance and good sliding properties, is observed that the evolution of the friction forces through time reveals a sliding without stick-slip with a relatively smooth steady state, even for low and moderate speeds. As can be seen from the pictured results in Figure 7, the kinetic tangential force has values between $0.1 \mathrm{~N}$ and $0.7 \mathrm{~N}$ for the longitudinal direction $\left(0^{\circ}\right)$, between $0.5 \mathrm{~N}$ and $1.2 \mathrm{~N}$ for the transversal direction $\left(90^{\circ}\right)$ and between $0.4 \mathrm{~N}$ and $0.8 \mathrm{~N}$ for the median direction $\left(45^{\circ}\right)$. It can be also observed a similarity of friction forces variations corresponding to the transverse and median sliding directions. Table 3 synthesises the static and kinetic Coulomb friction coefficients estimated by the ratio of the tangential force $F_{f}$ and the normal force $F_{n}$ along the three surface specimen directions: rolling or longitudinal (LD), transversal (TD) and median (DD) directions. 

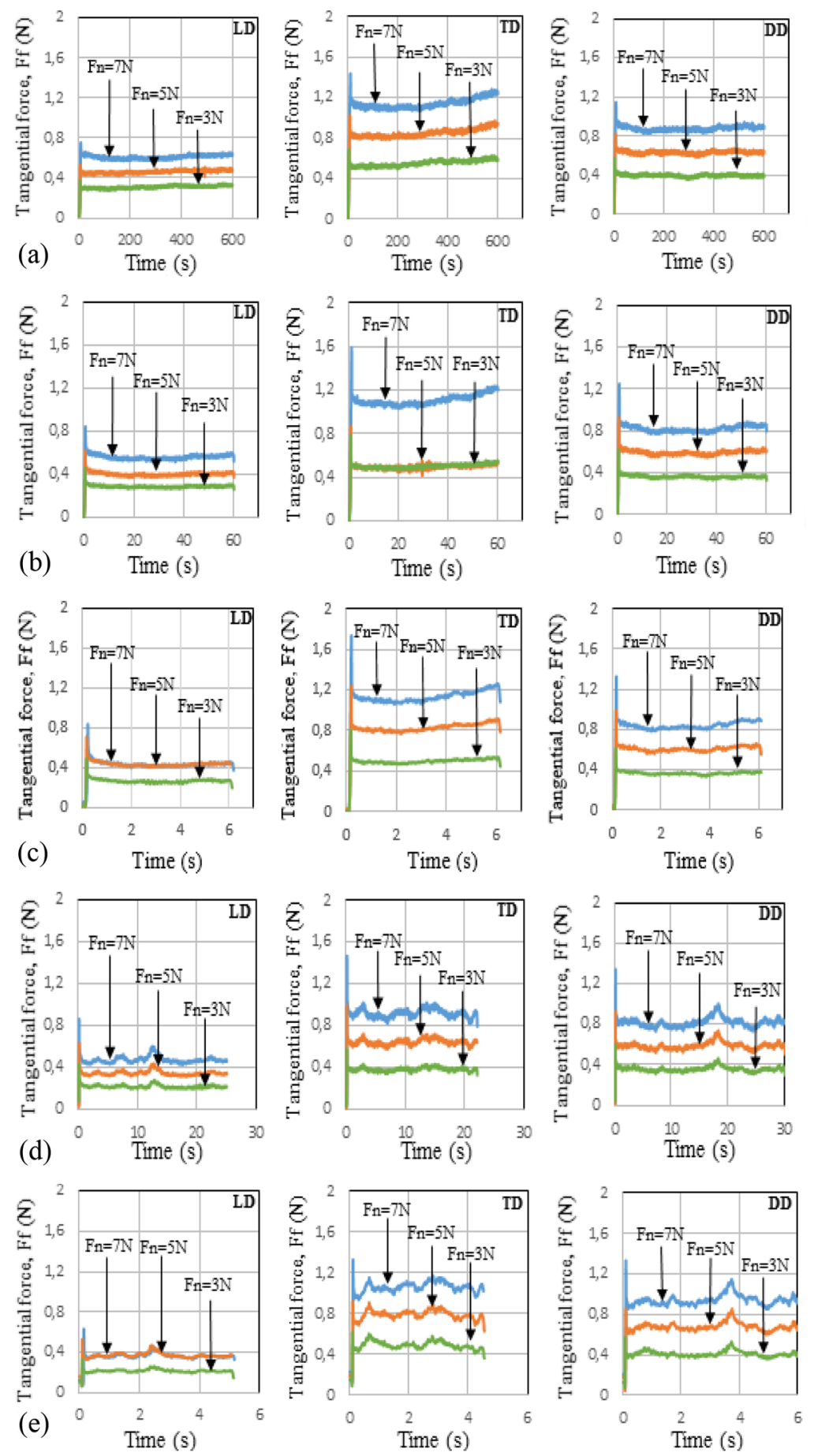

Fig. 7. Time variation of the tangential force for the longitudinal (LD), transversal (TD) and median (DD) directions. (a) $0.005 \mathrm{~mm} / \mathrm{s}$; (b) $0.05 \mathrm{~mm} / \mathrm{s}$; (c) $0.5 \mathrm{~mm} / \mathrm{s}$; (d) $1 \mathrm{~mm} / \mathrm{s}$; (e) $5 \mathrm{~mm} / \mathrm{s}$.

It can be observed that the sliding direction (longitudinal, transversal or median) influences the tangential forces $F_{f}$ and the friction coefficient.

The higher values occur for the direction orthogonal to the rolling direction (TD) and the smaller ones for the coefficient along the rolling direction (LD). So regarding the mean values of Coulomb coefficients these ones are around of $80 \%$ higher along the TD direction as compared to those of $\mathrm{LD}$ direction.
These results confirm the influence of the sliding stretching along the orthogonal direction to the longitudinal distribution of the surface asperities (Fig. 5). Similar phenomena have been highlighted by previous work of authors from analysis of micro-scratching tests along different directions using a diamond micro-blade [14]. It is then necessary to define an anisotropic friction law. Based on plastic theory applied to describe metallic materials behaviour, similar friction criteria can be defined using 
Table 3. Average values of Coulomb static and kinetic friction coefficient $\left(p_{c}-\right.$ contact pressure).

\begin{tabular}{|c|c|c|c|c|c|c|c|}
\hline \multirow{2}{*}{$\begin{array}{l}\text { Normal force } \\
F_{n}(\mathrm{~N})\end{array}$} & \multirow{2}{*}{$\begin{array}{l}\text { Sliding speed } \\
\mathrm{v}_{\mathrm{g}}(\mathrm{mm} / \mathrm{s})\end{array}$} & \multicolumn{3}{|c|}{ Coulomb static friction coefficient $\left(\mu_{s}\right)$} & \multicolumn{3}{|c|}{ Coulomb kinetic friction coefficient $\left(\mu_{k}\right)$} \\
\hline & & $\mathrm{LD}$ & TD & DD & $\mathrm{LD}$ & TD & DD \\
\hline \multirow{5}{*}{$\begin{array}{l}7 \\
\left(p_{c}=2.22 \mathrm{MPa}\right)\end{array}$} & 0.005 & 0.107 & 0.205 & 0.164 & 0.086 & 0.162 & 0.125 \\
\hline & 0.05 & 0.120 & 0.224 & 0.177 & 0.080 & 0.158 & 0.116 \\
\hline & 0.5 & 0.165 & 0.242 & 0.185 & 0.086 & 0.162 & 0.119 \\
\hline & 1 & 0.122 & 0.209 & 0.190 & 0.066 & 0.130 & 0.116 \\
\hline & 5 & 0.126 & 0.186 & 0.189 & 0.072 & 0.138 & 0.123 \\
\hline \multirow{5}{*}{$\begin{array}{l}5 \\
\left(p_{c}=1.59 \mathrm{MPa}\right)\end{array}$} & 0.005 & 0.106 & 0.205 & 0.165 & 0.092 & 0.170 & 0.126 \\
\hline & 0.05 & 0.123 & 0.284 & 0.184 & 0.080 & 0.165 & 0.118 \\
\hline & 0.5 & 0.140 & 0.242 & 0.193 & 0.086 & 0.164 & 0.121 \\
\hline & 1 & 0.126 & 0.198 & 0.185 & 0.068 & 0.128 & 0.117 \\
\hline & 5 & 0.104 & 0.181 & 0.170 & 0.073 & 0.143 & 0.124 \\
\hline \multirow{5}{*}{$\begin{array}{l}3 \\
\left(p_{c}=0.95 \mathrm{MPa}\right)\end{array}$} & 0.005 & 0.123 & 0.228 & 0.191 & 0.102 & 0.184 & 0.132 \\
\hline & 0.05 & 0.152 & 0.257 & 0.202 & 0.096 & 0.167 & 0.120 \\
\hline & 0.5 & 0.161 & 0.259 & 0.120 & 0.089 & 0.163 & 0.122 \\
\hline & 1 & 0.142 & 0.273 & 0.198 & 0.070 & 0.126 & 0.118 \\
\hline & 5 & 0.111 & 0.205 & 0.189 & 0.071 & 0.148 & 0.126 \\
\hline Mean values & & 0.133 & 0.238 & 0.176 & 0.089 & 0.166 & 0.122 \\
\hline
\end{tabular}

maximal work principle and normal rule of convex surface friction loci [15]. Regarding the plastic anisotropic Hill formulation (2) used to describe bulk anisotropic behaviour of AA2024-T351 aluminium alloy, in a similar way the following quadratic elliptic friction criterion $\Psi$ is chosen:

$$
\Psi(\vec{\tau})=\Psi\left(\tau_{x}, \tau_{y}\right)=G_{f} \tau_{x}^{2}+H_{f} \tau_{y}^{2}-\lambda^{2}=0 .
$$

Here $\tau_{x}, \tau_{y}$ represent the axis components of surface tangential stress $\vec{\tau}=\tau_{x} \overleftarrow{x}+\tau_{y} \vec{y}$ along the orthotropic plate axis $x$ (LD direction) and $y$ (TD direction). Taking into account the orientation angle $\alpha$ of friction shear stress $\vec{\tau}$, the corresponding two components along the orthotropic surface axis are defined by $\tau_{x}=\tau \cos (\alpha)$ and $\tau_{y}=\tau \sin (\alpha)$. Because the sliding direction $\vec{n}_{g}$ is defined by an angle $\theta$ in the $x y$ plane starting from the normal vector of the convex friction criterion i.e. $\vec{n}_{g}=-\vec{v}_{g} /\left\|\vec{v}_{g}\right\|=(\partial \Psi / \partial \vec{\tau})(\|\partial \Psi / \partial \vec{\tau}\|)$ it can be shown than $\vec{\tau} \cdot \vec{n}_{g}=\tau \cos (\alpha-\theta)$ and $\operatorname{tg}(\theta)=\left(H_{f} / G_{f}\right) \operatorname{tg}(\alpha)$. Taking into account the dependency of tangential stress with the contact pressure $p_{c}$ in terms of a Coulomb law it can be written $\vec{\tau} \cdot \vec{n}_{g}=\mu_{f}(\alpha) p_{c}=\mu_{f}(\theta) p_{c}, \tau(\theta)=\mu_{f}(\theta) p_{c}, \mu_{f}^{\prime}(\theta)=\mu_{f}(\theta) /$ $\cos (\alpha-\theta) \quad$ and $\quad \mu_{f}(\theta)=\sqrt{\left(\cos ^{2}(\theta) / G_{f}\right)+\left(\sin ^{2}(\theta) / H_{f}\right)}$ with $\tau=p_{c} / \sqrt{\left(\cos ^{2} \alpha / \mu_{x}^{2}\right)+\left(\sin ^{2} \alpha / \mu_{y}^{2}\right)}$. According to the friction values obtained for $\theta=0^{\circ}$ ( $\mathrm{LD}$ with $\alpha=0^{\circ}$ ) and $\theta=90^{\circ}$ (TD with $\alpha=90^{\circ}$ ), taking into account the local Coulomb friction law with $\tau\left(0^{\circ}\right)=\mu_{f}\left(0^{\circ}\right) p_{c}$ and $\tau\left(90^{\circ}\right)=$ $\mu_{f}\left(90^{\circ}\right) p_{c}$ it is easy to verify from (3) that $G_{f}=1 / \mu_{f}^{2}\left(0^{\circ}\right)$, $H_{f}=1 / \mu_{f}^{2}\left(90^{\circ}\right)$ and $\lambda=p_{c}$. Consequently the anisotropic friction law can be written in the following form:

$$
\begin{aligned}
& \left(\tau_{x}^{2} / \mu_{f}^{2}\left(0^{\circ}\right)\right)+\left(\tau_{y}^{2} / \mu_{f}^{2}\left(90^{\circ}\right)\right)=p_{c}^{2} \text { with } \\
& \mu_{f}(\theta)=\sqrt{\mu_{f}^{2}\left(0^{\circ}\right) \cos ^{2}(\theta)+\mu_{f}^{2}\left(90^{\circ}\right) \sin ^{2}(\theta)}
\end{aligned}
$$

where the orientation of tangential stress, defined by the angle $\alpha$, is estimated by:

$$
\operatorname{tg}(\alpha)=\left(\mu_{f}\left(90^{\circ}\right) / \mu_{f}\left(0^{\circ}\right)\right) \operatorname{tg}(\theta) .
$$

Regarding from Table 3 the mean values of contact pressure $\left(p_{c}=1.58 \mathrm{MPa}\right)$ and of friction coefficients, in the case of the sliding along the median direction DD $\left(\theta=45^{\circ}\right)$ the corresponding friction coefficients for both static and kinematics ones $\left(\mu_{s}\right.$ and respectivelly $\mu_{k}$ ) have a magnitude close to the root mean square of LD and TD values $(0.193$ for static friction and 0.133 for kinetic friction, respectively $9 \%-9.5 \%$ error estimation) with a shear stress orientation $\alpha \approx 60^{\circ}$ (Fig. 8).

Using the previous anisotropic friction model analysis it is necessary to distinguish the values of the Coulomb coefficients $\mu_{f}(\theta)$ determined from the measurement of tangential stress component in the direction of sliding and the friction coefficients $\mu_{f} \prime(\theta)$ estimated from the measurement of the resulting tangential stress vector. From the experimental point of view, this problem is still difficult to control because the results will be functions of the type of load sensors used by tribometers. On the other hand, if $\cos (\alpha-\theta) \approx 1$ corresponding to $\alpha-\theta$ less than $15^{\circ}$ it can be neglected the influence of the tangential force component type measurement during 


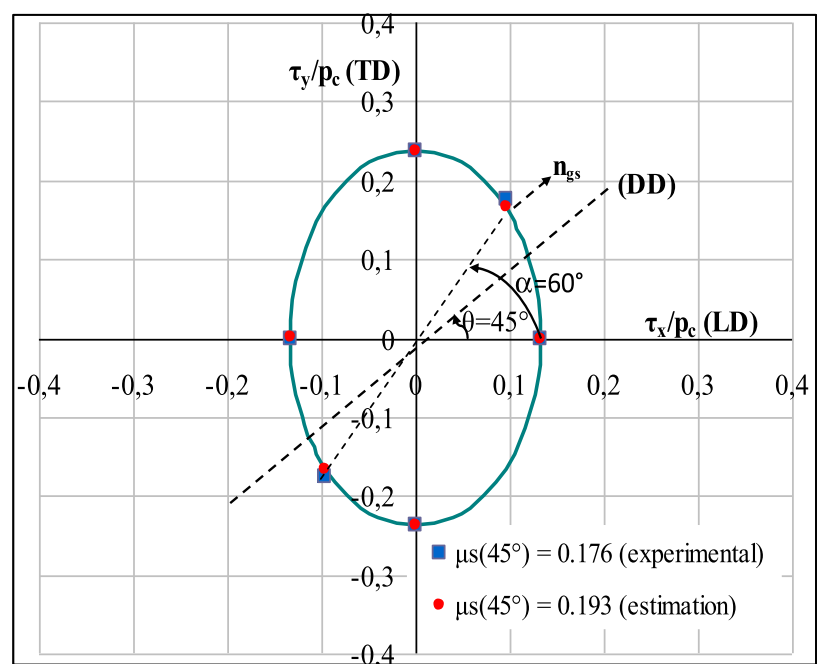

(a)

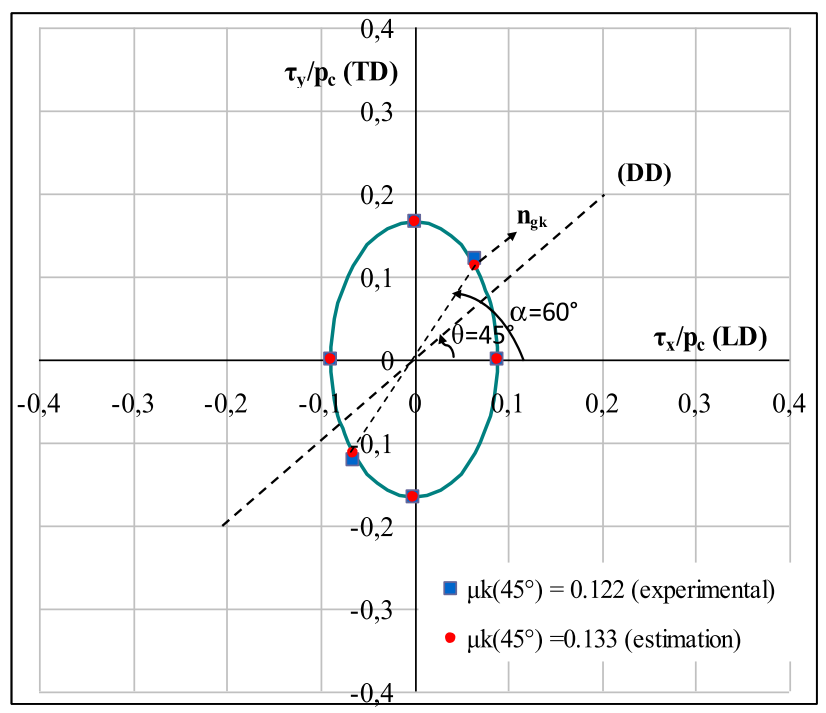

(b)

Fig. 8. Elliptic anisotropic friction criterion concerning rolled AA2024-T351/UHMWPE polyethylene sliding contact; (a) static friction; (b) kinetic friction.

the sliding tests along different directions on the estimation of Coulomb coefficient values (maximum estimation error of $3.5 \%$ ).

Consequently the above expressions (4) describe with a good approximation the observed anisotropic surface friction behaviour. Concerning the sliding velocities and the contact pressures these mechanical contact variables have relatively small influences $(10 \%-15 \%)$ on the estimation of the friction coefficients. A weak maximum peak is reached especially for a sliding speed of $0.5 \mathrm{~mm} / \mathrm{s}$.

\section{Tribological fractal analysis}

Surface roughness has a large impact on the tribological phenomena such as contact mechanics, friction, wear, lubrication, etc. When two flat surfaces are in contact, surface topography causes discrete contact points. The mode of surface deformation may be elastic, elastic-plastic or plastic and depends on surface topography, mechanical and physical material proprieties, normal and shear forces or stresses. Fractal geometry method has been widely used in recent years and can be applied to characterize surface topography and contacts mechanics $[16,17]$. According to the previous work of authors concerning fractal surface geometry analysis of the anisotropic AA2024-T351 thick plate [13], the purpose of the study is to investigate the fractal character of the friction coefficient evolution during the transition between the static state and the kinetic one in the case of a sliding process between a polyethylene cylindrical pin and the rolled AA2024-T351 surface plate. It is then proposed to analyse the friction curves of Figure 7 corresponding to the higher contact pressure $p_{c}=2.22 \mathrm{MPa}$ (constant normal force $F_{n}=7 \mathrm{~N}$ ) and the chosen constant five speeds. The fractal dimension of friction coefficient variation $D_{\mu}$ can be computed using the following equations $[16,17]$ :

$$
D_{\mu}=\frac{4-D_{s}}{2}, 0<D_{s}<2
$$

The slope of the structure function $D_{s}$, plotted on double logarithmic coordinates, is estimated by using three points defined by $x_{f_{s}}=\left\{x_{f_{s} 1}, x_{f s 2}, x_{f s 3}\right\}, y_{f s}=\left\{y_{f s 1}, y_{f_{s} 2}, y_{f s 3}\right\}$ :

$$
D_{s}=\operatorname{slope}\left(x_{f s}, y_{f s}\right)
$$

This slope is a measure of the steepness of a line, or a section of a line, connecting two points and is computed by finding the ratio $m$ of the "vertical change" to the "horizontal change" between (any) two distinct points on a line i.e. $m=\Delta y / \Delta x$. The corresponding friction structure function $S_{\mu}$ can be written as [17-19]:

$$
S_{\mu}(N, k)=\frac{1}{N-k} \sum_{i=1}^{N-k}\left(y_{i+k}-y_{i}\right)^{2} .
$$

Here $k$ is the increment of $x$ ordinate, $k=1, \ldots, N-1$ and $N$ is the length of the $y$ vector; here $N=2000$, except the tests for $0.5 \mathrm{~mm} / \mathrm{s}$ where $N=500$ and $1 \mathrm{~mm} / \mathrm{s}$ where $N=400$. The straight line that approximates the friction structure function $S_{\mu}$ can be determined in function of $x_{f}=0,0.01, \ldots, 2$. as following:

$$
y_{f}\left(x_{f}\right)=D_{s} \cdot x_{f}+y_{f s 1}-D_{s} \cdot x_{f s 1} .
$$

Figures 9-13 describe the $\log -\log$ curves (friction structure function - correlation length) and the corresponding straight line $\left(y_{f}\left(x_{f}\right)\right.$, where $\left(y_{f}=\log \left(S_{\mu}(N, k)\right.\right.$ and $x_{f}=$ $\log (k))$ that approximates the friction structure function variation of measurements along the three directions (LD, TD and DD).

From the plotted log-log curves and the corresponding straight line $\left(y_{f}\left(x_{f}\right)\right)$ it can be observed that the friction coefficient evolution, especially during the transition between static and kinetic sliding, reveals a fractal character up to 20 time's increment point (around a sliding distance of $0.025 \mathrm{~mm}$ ) for $v=0.5 \mathrm{~mm} / \mathrm{s}, v=1 \mathrm{~mm} / \mathrm{s}, v=5 \mathrm{~mm} / \mathrm{s}$ and up to 80 time's increment point (around a sliding distance of $0.150 \mathrm{~mm}$ ) for $v=0.05 \mathrm{~mm} / \mathrm{s}$. For the lowest speed 

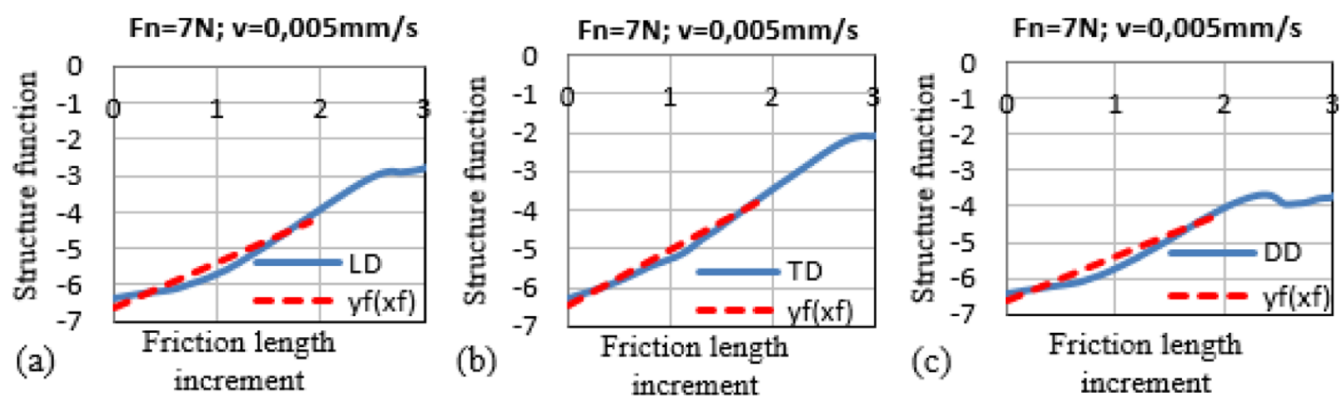

Fig. 9. $\log -\log$ curves (structure function-correlation length) and the straight line $\left(y_{f}\left(x_{f}\right)\right)$ for $F_{n}=7 \mathrm{~N}$ and $v=0.005 \mathrm{~mm} / \mathrm{s}$; (a) longitudinal direction (LD); (b) transversal direction (TD); (c) median direction (DD).
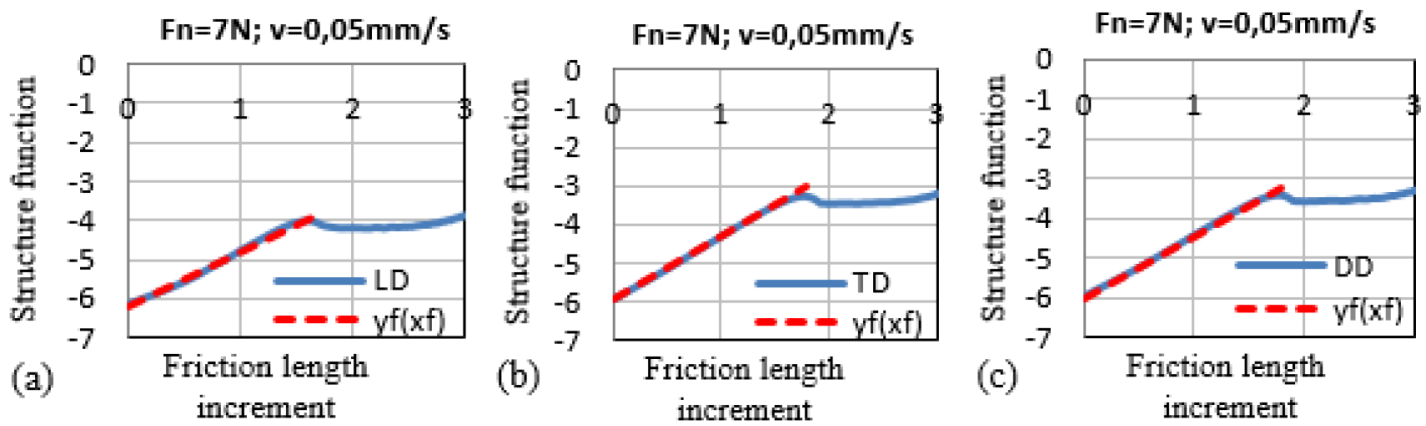

Fig. 10. $\log -\log$ curves (structure function-correlation length) and the straight line $\left(y_{f}\left(x_{f}\right)\right)$ for $F_{n}=7 \mathrm{~N}$ and $v=0.05 \mathrm{~mm} / \mathrm{s}$; (a) longitudinal direction (LD); (b) transversal direction (TD); (c) median direction (DD).
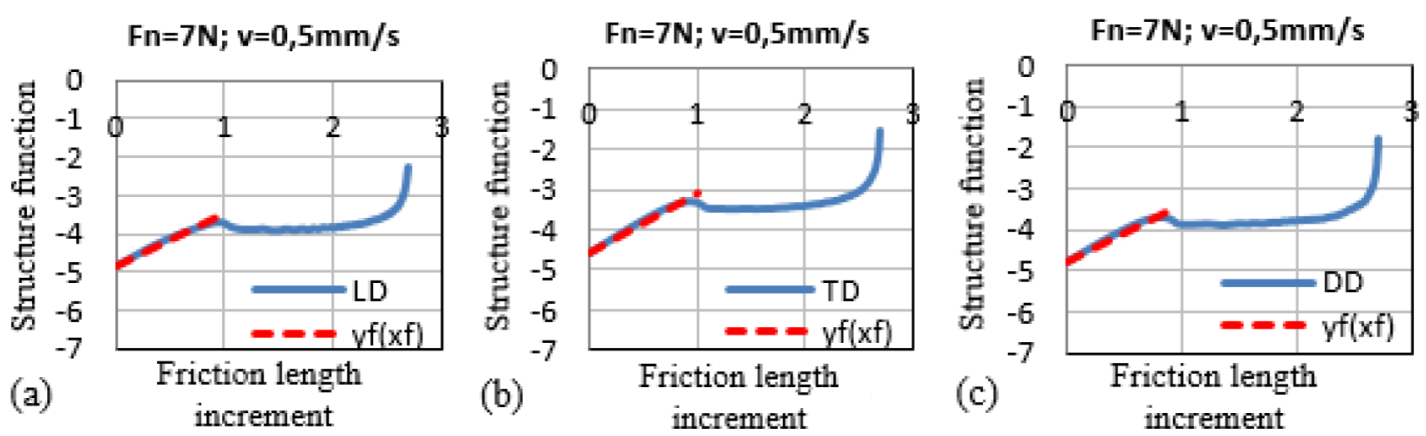

Fig. 11. $\log -\log$ curves (structure function-correlation length) and the straight line $\left(y_{f}\left(x_{f}\right)\right)$ for $F_{n}=7 \mathrm{~N}$ and $v=0.5 \mathrm{~mm} / \mathrm{s}$; (a) longitudinal direction (LD); (b) transversal direction (TD); (c) median direction (DD).

$\mathrm{Fn}=7 \mathrm{~N} ; \mathrm{v}=1 \mathrm{~mm} / \mathrm{s}$

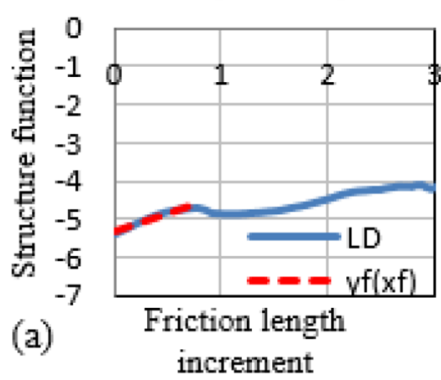

$\mathrm{Fn}=7 \mathrm{~N} ; \mathrm{v}=1 \mathrm{~mm} / \mathrm{s}$

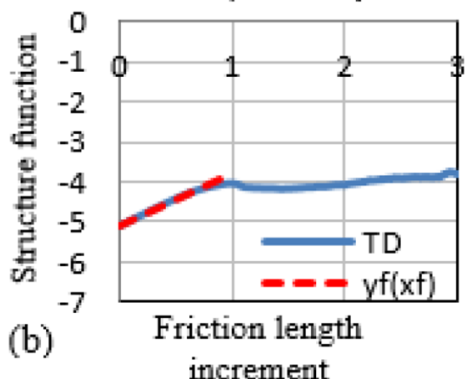

$\mathrm{Fn}=7 \mathrm{~N} ; \mathrm{v}=1 \mathrm{~mm} / \mathrm{s}$

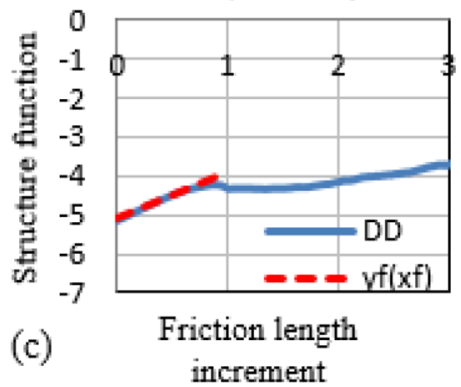

Fig. 12. Log-Log curves (structure function-correlation length) and the straight line $\left(y_{f}\left(x_{f}\right)\right)$ for $F_{n}=7 \mathrm{~N}$ and $v=1 \mathrm{~mm} / \mathrm{s}$; (a) longitudinal direction (LD); (b) transversal direction (TD); (c) median direction (DD). 

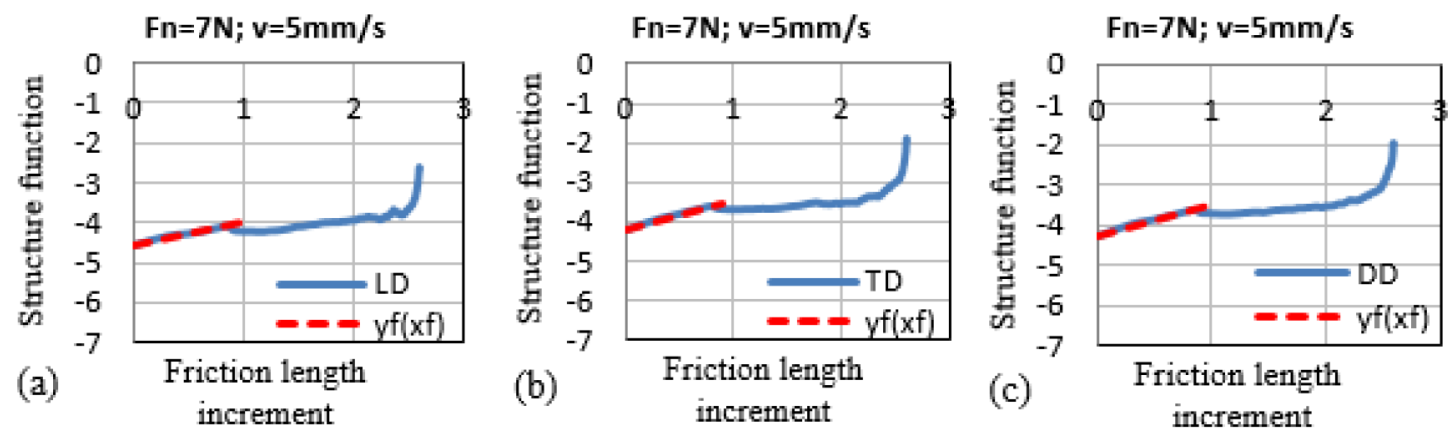

Fig. 13. $\log -\log$ curves (structure function-correlation length) and the straight line $\left(y_{f}\left(x_{f}\right)\right)$ for $F_{n}=7 \mathrm{~N}$ and $v=5 \mathrm{~mm} / \mathrm{s}$; (a) longitudinal direction (LD); (b) transversal direction (TD); (c) median direction (DD).

Table 4. Friction fractal dimension values for $v=0.005 \mathrm{~mm} / \mathrm{s}, v=0.05 \mathrm{~mm} / \mathrm{s}$ and $v=0.5 \mathrm{~mm} / \mathrm{s}\left(F_{n}=7 \mathrm{~N}\right)$.

\begin{tabular}{|c|c|c|c|c|c|c|c|c|}
\hline \multicolumn{9}{|c|}{ Friction fractal dimension, $D_{\mu}$} \\
\hline \multicolumn{3}{|c|}{$F_{n}=7 \mathrm{~N} ; v=0.005 \mathrm{~mm} / \mathrm{s}$} & \multicolumn{3}{|c|}{$F_{n}=7 \mathrm{~N} ; v=0.05 \mathrm{~mm} / \mathrm{s}$} & \multicolumn{3}{|c|}{$F_{n}=7 \mathrm{~N} ; v=0.5 \mathrm{~mm} / \mathrm{s}$} \\
\hline LD & $\mathrm{TD}$ & $\mathrm{DD}$ & $\mathrm{LD}$ & $\mathrm{TD}$ & $\mathrm{DD}$ & $\mathrm{LD}$ & $\mathrm{TD}$ & DD \\
\hline * & $*$ & $*$ & 1.305 & 1.202 & 1.226 & 1.384 & 1.284 & 1.393 \\
\hline
\end{tabular}

Table 5. Friction fractal dimension values for $v=1 \mathrm{~mm} / \mathrm{s}, v=5 \mathrm{~mm} / \mathrm{s}\left(F_{n}=7 \mathrm{~N}\right)$.

Fiction fractal dimension, $D_{\mu}$

\begin{tabular}{lllllll}
\hline & \multicolumn{2}{c}{$F_{n}=7 \mathrm{~N} ; v=1 \mathrm{~mm} / \mathrm{s}$} & & & \multicolumn{3}{c}{$F_{n}=7 \mathrm{~N} ; v=5 \mathrm{~mm} / \mathrm{s}$} \\
\cline { 5 - 7 } $\mathrm{LD}$ & $\mathrm{TD}$ & $\mathrm{DD}$ & & $\mathrm{LD}$ & TD & DD \\
\hline 1.526 & 1.346 & 1.406 & & 1.716 & 1.623 & 1.605 \\
\hline
\end{tabular}

$(v=0.005 \mathrm{~mm} / \mathrm{s})$ the friction coefficient variation doesn't have a fractal character $(*)$.

The corresponding computed values of the friction fractal dimension $D_{\mu}$ are given in Tables 4 and 5 .

As can be shown in Figure 14 the computed friction fractal dimension increases with the speed and have relatively similar values for TD and DD directions (except for the intermediate sliding speed of $0.5 \mathrm{~mm} / \mathrm{s}$ ). The higher values are obtained for a sliding along the rolling direction $\mathrm{LD}$ and the smallest ones for the transversal direction TD. All these friction fractal information confirm again the friction and surface anisotropy together with the conclusion than concerning the estimation of a steady state kinetic friction it is sufficient to use a measuring range of tangential forces for a maximal sliding distance between 0.025 and $0.150 \mathrm{~mm}$. Furthermore the computed friction fractal dimension $D_{\mu}$ can be used to estimate an average ratio between the static and kinetic friction coefficients.

\section{Conclusions}

The main purpose of this scientific study has been to evaluate the influence of material anisotropy on the sliding of an ultra-high-molecular-weight polyethylene (UHMWPE) cylindrical pin over an aluminium alloy AA2024-T351 rolled thick plate in the range of low and moderate sliding speeds. Starting from previous works

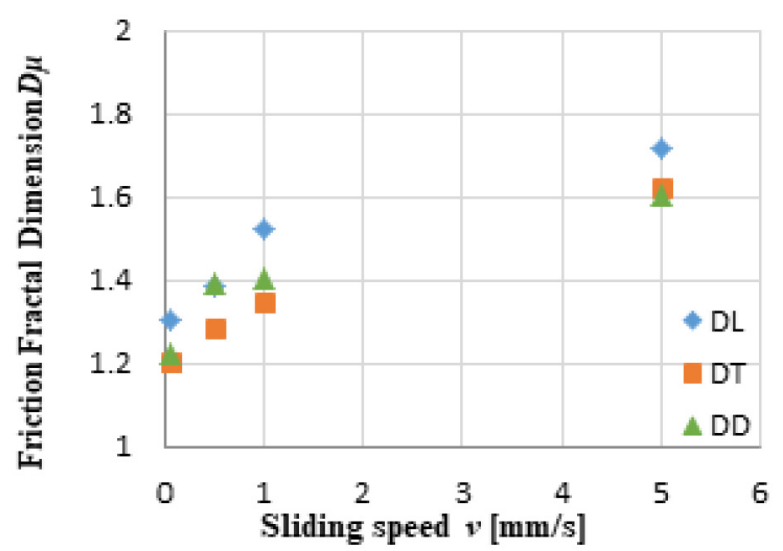

Fig. 14. Variation of friction fractal dimension with the sliding speed corresponding to the three different directions: longitudinal (DL or LD), transversal (DT or TD) and median (DD).

rheological elastic and plastic parameters of an anisotropic plastic Hill criterion was presented to describe and understand the bulk anisotropy of studied aluminium rolled plate in order to have a reliable estimation of the stress state at the contact interfaces. To characterise surface friction properties a lot of dry sliding tribological tests have been performed using the motion of a polyethylene cylindrical pin along a linear trajectory $(3 \mathrm{~mm})$ under a 
constant normal force $(3 \mathrm{~N}, 5 \mathrm{~N}, 7 \mathrm{~N})$ and at constant sliding speeds $(0.005,0.05,0.5,1,5 \mathrm{~mm} / \mathrm{s})$. All the experimental tests have been realized along three directions: a longitudinal one $\left(\mathrm{LD}\right.$ or $\left.0^{\circ}\right)$, corresponding to the rolling direction of the sample, a transversal one (TD or $90^{\circ}$ ), which represents the direction perpendicular to the rolling direction, and a median direction (DD or $45^{\circ}$ ). According to the obtained results it can be concluded that the rolling AA2024-T351 thick plate has anisotropic tribologic properties. So the sliding direction influences the friction coefficient: a smallest coefficient value occurs for the longitudinal direction, a higher one for the transversal direction and a root square mean value for the median direction. It has been also observed that Coulomb coefficients have a relatively small variation with the sliding speeds and contact pressure and reaching maximum values generally for a sliding speed of $0.5 \mathrm{~mm} / \mathrm{s}$. A quadratic elliptic function has been identified to describe the observed anisotropic surface friction with an average error around of $10 \%$. Due to the nature of the polyethylene surface properties the variation of the friction force or of the Coulomb friction coefficient versus time does not describe a stick-slip sliding generally characterising small and very small sliding speeds. Concerning the analysis of the transitions between the static friction and the steady sliding state, the investigation of the times' friction coefficient evolution reveals a fractal character. The log-log curves (structure function-correlation length), the straight line $\left(y_{f}\left(x_{f}\right)\right)$ and the fractal dimension show a friction fractal character up to $0.025-0.150 \mathrm{~mm}$ sliding distance, except for the very small sliding speed $(0.005 \mathrm{~mm} / \mathrm{s})$. Differences of fractal friction dimensions along the orthotropic material directions confirm the anisotropic tribological properties observed by the material surface topography and the Coulomb friction coefficient estimation.

Acknowledgements. The presented scientific article concerns a research work realized during the $\mathrm{PhD}$ Thesis of Elisabeta PIRVA under an international collaboration agreement between University Politehnica of Bucharest - UPB (Co-Supervisor Prof. Andrei TUDOR) and National Institute of Applied Sciences of Rennes INSA Rennes (Co-Supervisor Assoc. Prof. Hab. Adinel GAVRUS) with scientific guidance and experimental support of Prof. Sorin CANANAU (UPB) and Ass. Prof. Andrei STOICA (UPB). The authors thank the ERASMUS Program, Rennes Metropole (France) and UPB Doctoral School's Directors Boards (Romania) for the assigned grants necessary to ensure the required mobility of the $\mathrm{PhD}$ Student and of the above involved researchers.

\section{References}

[1] E. Gustafsson, Investigation of friction between plastic parts, $\mathrm{PhD}$ thesis, Chalmers University of Technology, Göteborg, Sweden, 2013

[2] P. Menezes, S. Ingole, M. Nosonovsky, S. Kailas, M. Lovell, Tribology for scientists and engineers, from basics to advanced concepts, Springer, Berlin (2013) pp. 43-44
[3] R.P. Nachane, G.F.S. Haussain, K.R. Krishana Iyer, Theory of the stick-slip effect in friction, Indian J. Fib. Textile Res. 23, 201-208 (1998)

[4] J. Takadoum, Materials and surface engineering in tribology, John Wiley \& Sons, Hoboken, NJ, 2007, pp. 63-64., first published by Hermes Science/Lavoisier entitles: "Matériaux et surfaces en tribology"

[5] N.A. Stoica, A. Tudor, Some aspects concerning the behaviour of friction materials at low and very low sliding speeds, Tribol. Ind. 37, 374-379 (2015)

[6] S.S. Antoniu, A. Cameron, The friction-speed relation for stick data, Wear 36, 235-254 (1976)

[7] E. Cazimitrovici, M.V. Suciu, Laminarea Materialelor Metalice Speciale, Editura BREN, Bucharest, Romania, 2000

[8] Generic Material Properties of Ultra High Molecular Weight Polyethylene (UHMWPE) http://www.dielectriccorp.com/ downloads/thermoplastics/uhmw.pdf

[9] E. Pirva, Etudes mécaniques et multi-échelle du comportement isotrope et anisotrope $3 \mathrm{D}$ en grandes déformations plastiques. Application à la modélisation numérique de l'emboutissage profonde des tôles des alliages d'aluminium, Research Master's Thesis, Supervisor: Assoc. Prof. Adinel GAVRUS, INSA Rennes, France, 2014

[10] W. Nasri, A. Gavrus, A. Kouadri-David, K. Sai, Applications of multi-scale models to numerical simulation and experimental analysis of anisotropic elastoplastic behavior of metallic sheets, Key Eng. Mater. 611-612, 536-544 (2014)

[11] W. Nasri, A. Gavrus, A. Kouadri-David, K. Sai, Experimental characterization and numerical modeling of threedimensional anisotropic behavior of a thick sheet aluminum alloy AA2024-T351 using multi-scale approaches, WIT Trans. Built Environ. 166, 163-177 (2016)

[12] Manual of UMT Multi-Specimen Test System Hardware Ind. \& Applications Available at http://erc.ncat.edu/Facilities/ Manuals/CETR_UMT2_Manual.pdf

[13] E. Pirva, A. Tudoror, A. Gavrus, Fractal analysis of surface micro-topography for a rolled anisotropic thick sheet of aluminium alloy (AA2024-T351), IOP Conf. Ser. Mater. Sci. Eng. 147, 1 (2016)

[14] E. Pirva, A. Tudor, A. Gavrus, G. Chisiu, N. Stoica, A. Predescu, Micro-scratching tests of a rolled aluminium alloy AA2024-T351 thick plate using a diamond micro-blade, IOP Confer. Ser. Mater. Sci. Eng. 174, 1 (2016)

[15] P. Montmitonnet, F. Delamare, E. Felder, N. Marsault, Cours de Tribologie de la Mise en Forme, Master/DEA, Ed. Ecole Nationale des Mines de Paris, France 1992

[16] H. Zahouani, R. Vargiolu, J.L. Loubet, Fractal Models of Surface Topography and Contact Mechanics, Math. Comp. Model. 28, 517-534 (1998)

[17] B. Bhushan, Contact mechanics of rough surfaces in tribology: single asperity contact, Appl. Mech. Rev. 49, 275-297 (1996)

[18] Y. Morag, I. Etsion, Resolving the contradiction of asperities plastic to elastic mode transition in current contact models of fractal rough surfaces, Wear 262, 624-629 (2007)

[19] B.B. Mandelbrot, The fractal geometry of nature, Phys. Scr. 32, 257-260 (1982)

Cite this article as: E. Pirva, A. Tudor, A. Gavrus, N. Stoica, S. Cananau, Some aspects regarding the influence of the anisotropy of an AA2021-T351 rolled thick plate on its tribological behaviour, Mechanics \& Industry 20, 610 (2019) 